\title{
Reversal of Roux-en-Y Gastric Bypass Fails to Facilitate the Management of Recalcitrant Hypocalcaemia Caused by Primary Hypoparathyroidism
}

Katrien Corbeels, Msc ${ }^{1}$; Nele Steenackers, $\mathrm{Msc}^{1}$; Matthias Lannoo, MD, $\mathrm{PhD}^{1}$; Ann Mertens, $\mathrm{MD}, \mathrm{PhD}^{1}$; Ellen Deleus, MD ; Nelson Cunha, $\mathrm{MD}^{2}$; Pieter Sinonquel, MD³; Christophe Matthys, $\mathrm{PhD}^{1}$; Ann Meulemans, $\mathrm{PhD}^{1}$; Roman Vangoitsenhoven, MD, $\mathrm{PhD}^{1}$; Bart Van der Schueren, MD, $\mathrm{PhD}^{1}$

1 Department of Chronic diseases, Metabolism \& Ageing (CHROMETA), Clinical and Experimental Endocrinology, KU Leuven, O\&N1 Herestraat 49 - box 902, 3000 Leuven, Belgium; ${ }^{2}$ Department of Endocrinology, Coimbra Hospital and University Centre (CHUC), Coimbra, Portugal; ${ }^{3}$ Department of Translational Research in Gastrointestinal Disorders (TARGID), KU Leuven, Leuven, Belgium.

Corresponding author: Katrien Corbeels katrien.corbeels@kuleuven.be

Conflict of Interest: The authors declare that they have no conflicts of interest.

Keywords: Bariatric surgery; Roux-en-Y gastric bypass; Thyroidectomy; Parathyroid hormone; Calcium

Funding sources: This work was supported by the FWO (Flemish Research Council) 1 S27317N and $1802714 \mathrm{~N}$.

\section{ABSTRACT}

Roux-en-Y gastric bypass (RYGB) is thought to reduce calcium absorption from the gut. Here, we report the case of a patient with a RYGB, who developed primary hypoparathyroidism after a total thyroidectomy, leading to recalcitrant hypocalcaemia. Despite aggressive oral calcium and calcitriol supplementation, she remained hypocalcaemic and required intravenous (IV) calcium supplementation to control her symptoms, and to keep calcium serum levels within an acceptable range. Teriparatide treatment improved calcium levels marginally. This treatment, however, was poorly tolerated and ultimately stopped by the patient. As a last resort, reversal of RYGB was performed to improve calciumabsorption from the gut. Unfortunately, IV calcium supplementation remained necessary. This case illustrates that the reversal of RYGB is not always a guarantee for success in managing recalcitrant hypocalcaemia.

\section{INTRODUCTION}

As the prevalence of obesity increased worldwide, so did the amount of patients that underwent bariatric surgery [1, 2]. In general, bariatric surgery is beneficial in patients with morbid obesity, as it leads to sustained weight loss and improvement or even remission of many of the obesityrelated co-morbidities [3]. Even before surgery, patients with obesity are often vitamin D 
deficient and suffer from secondary hyperparathyroidism (SHPT), which compromises bone health [4]. After bariatric surgery, SHPT can aggravate to compensate for impaired intestinal calcium absorption [4]. Here, we report the case of a patient with a previous RYGB, who developed hypocalcaemia because of her inability to raise PTH levels following a total thyroidectomy complicated by postsurgical hypoparathyroidism.

\section{CASE REPORT}

A 41-year-old female patient was referred to our hospital in July 2018 for persisting hypocalcaemia. In 2006, the patient had undergone an adjustable gastric banding (with a preoperative body mass index (BMI) of $47 \mathrm{~kg} / \mathrm{m} 2$ ). Two years later, a secondary sleeve gastrectomy (SG) was performed given continued weight gain up to BMI $52 \mathrm{~kg} / \mathrm{m} 2$. The SG was converted into a Roux-en-Y gastric bypass (RYGB) in 2014 (at BMI $50 \mathrm{~kg} / \mathrm{m} 2$ ) due to weight regain after an initial decrease. In June 2017, a total thyroidectomy was performed for the presence of a cold nodule of 39 by 23 by $19 \mathrm{~mm}$ in the right thyroidal lobe. Preoperative serum calcium, phosphate, magnesium and 25-hydroxyvitamin $\mathrm{D}(25(\mathrm{OH}) \mathrm{D})$ levels were within the normal range, while PTH was moderately elevated (224 ng/L, normal range: 14.9-56.9 $\mathrm{ng} / \mathrm{L})$. Histological evaluation of the resected thyroid gland and thyroidal nodule showed no malignancy. No parathyroid glands were found in the resection specimen. Postoperative evaluation showed a low PTH $(<6 \mathrm{ng} / \mathrm{L})$ indicating that the parathyroid glands had been severed during the thyroidectomy and as a result primary hypoparathyroidism developed. When referred to our out-patient clinic in 2018, the patient presented with hypocalcaemia (serum calcium of $7.08 \mathrm{mg} / \mathrm{dL}$, normal range: 8.6-10.2), a normal serum phosphate of 3.47 $\mathrm{mg} / \mathrm{dL}$ (normal range: $2.5-4.5 \mathrm{mg} / \mathrm{dL}$ ), normal serum levels of $25(\mathrm{OH}) \mathrm{D}$ and 1,25hydroxyvitamin $\mathrm{D}(1,25(\mathrm{OH}) 2 \mathrm{D})$ and symptoms of paraesthesia and muscle cramps in both hands, despite high doses of calcium and activated vitamin D oral substitution. Serum PTH level was $29.5 \mathrm{ng} / \mathrm{L}$, which is inadequately low. The patient was hospitalised and treated with high doses of oral calcium citrate $(4 \times 6000 \mathrm{mg}$ daily), oral calcitriol $(3 \times 2 \mu \mathrm{g}$ daily), colecalciferol (300,000 IU single administration), chlorthalidon $(1 \times 50 \mathrm{mg}$ daily $)$ and intravenous (IV) calcium gluconate (4500 mg in $24 \mathrm{~h}$ ) to restore serum calcium levels. Due to an inadequate response to oral and IV therapy, daily subcutaneous administration of $20 \mu \mathrm{g}$ of 
teriparatide, a recombinant protein form of PTH, was added to the treatment. After 1 week, symptoms improved and serum calcium levels increased up to $8.4 \mathrm{mg} / \mathrm{dL}$. This treatment, however, had to bestopped because of headaches, fatigue and myalgia. Therefore, IV calcium remained necessary twice a week. Given the persistent need of IV therapy, a reversal of the RYGB was performed since we hypothesized an insufficient intestinal calcium absorption as underlying cause of the recalcitrant hypocalcaemia. Unfortunately, hypocalcaemia persisted with serum calcium levels of $7.5 \mathrm{mg} / \mathrm{dL}$ and IV therapy, dose-dependent on serum calcium level, remained necessary despite the reversal of the RYGB. No other postoperative complications occurred after the reversal. The patient did however start to regain weight.

\section{DISCUSSION}

In this case, we report a patient who, following a total thyroidectomy, developed primary hypoparathyroidism that resulted in recalcitrant hypocalcaemia. After thyroidectomy, the prevalence of hypoparathyroidism ranges between 10 and 48\% [5]. To maintain serum calcium, dietary calcium is absorbed in the duodenum and proximal jejunum via a combination of active and passive paracellular transport, both dependent on 1,25(OH)2D [4]. After RYGB, the anatomical alterations are believed to lead to calcium and vitamin $D$ hypoabsorption through a bypass of the major area of nutrient absorption, reduced gastric acid secretion and altered intestinal transit $[4,6]$. In case of decreasing serum calcium levels, the parathyroid glands will secrete PTH. Subsequently, PTH will stimulate the release of calcium from the bone to maintain normal serum calcium levels and activate vitamin $D$ to stimulate intestinal absorption and renal calcium reabsorption [7]. SHPT is believed to arise as a compensatory mechanism for the diminished calcium uptake from the gut, as the duodenum is bypassed [4]. Hence, a total thyroidectomy could interfere with this compensatory process, resulting in an increased risk of postoperative primary hypoparathyroidism and hypocalcaemia [8]. Off-label treatment with teriparatide was started in our patient and resulted in stable serum calcium levels after 1 week. Unfortunately, the treatment had to be discontinued because of side effects. Reversal of RYGB has been performed previously in similar cases in an effort to restore the intestinal calcium uptake $[9,10]$. Therefore, we decided when all other treatments had failed that a reversal of the RYGB was warranted. Nonetheless, the patient did not have a sustained increase in serum calcium and remained dependent on IV calcium. Previous case reports suggest that patients with preceding gastric bypass have an increased risk of severe hypocalcaemia after total thyroidectomy [11]. A study by Droeser et al., however, did not find a higher risk for hypocalcaemia after total thyroidectomy in patients after RYGB [11], 
supporting the notion that RYGB is not responsible for the hypocalcaemia developed as a result of postsurgical hypoparathyroidism. To our knowledge, we are the second case to report the offlabel use of teriparatide in a patient with severe hypocalcaemia after RYGB and total thyroidectomy [9]. The administration of teriparatide was unable to reverse hypocalcaemia, and a surgical reversal of RYGB was advised to increase intestinal calcium absorption. In this case, the reversal of RYGB treated the hypocalcaemia successfully. In our patient, however, severe hypocalcaemia persisted after reversal, requiring continued IV calcium therapy. In hindsight, it might have been more prudent to insert a gastrostomy tube into the gastric remnant to administer calcium and calcitriol and assess the absorption [12-14]. This would have enabled us to investigate whether a better calcium absorption could be achieved by reusing the excluded limb. If calcium absorption would have improved, this would have been in favour of the reversal of RYGB to improve calcium status. In the absence thereof, it would have been a warning that our attempts to normalize serum calcium by reversing the RYGB may be unsuccessful. Another option would be to use the recombinant form of PTH (Natpar®) indicated for patients with chronic hypoparathyroidism. However, this was unavailable at the time of the study. These findings support the notion that calcium hypoabsorption was not the primary cause of hypocalcaemia in our patient and that reversal of the RYGB was thus not useful in managing this case of postsurgical hypoparathyroidism resulting in severe hypocalcaemia. It is important to carefully way the consequences of reversing a RYGB for recalcitrant hypocalcaemia caused by primary hypoparathyroidism, as reduced absorption of calcium from 


\section{REFERENCES}

1. World Health Organization. [accessed November 28 2019]. Fact sheet: Obesity and Overweight 2018. http://www.who.int/mediacentre/factsheets/fs311/en/.

2. American Society for Metabolic and Bariatric Surgery. [Accessed November 28 2019] Fact sheet: metabolic and bariatric surgery 2013. http://asmbs.org/.

3. Swinburn BA, Kraak VI, Allender S, et al. The global syndemic of obesity, undernutrition, and climate change: the lancet commission report. Lancet. 2019;393(10173):791-846.

4. Corbeels $\mathrm{K}$, Verlinden $\mathrm{L}$, Lannoo $\mathrm{M}$, et al. Thin bones: vitamin $\mathrm{D}$ and calcium handling after bariatric surgery. Bone Rep. 2018;8:57-63.

5. Meija M, Gonzalez-Devia D, Fierro F, et al. Hypocalcemia posthyroidectomy: prevention, diagnosis and management. J Transl Sci. 2018;4(2):1-7.

6. Hewitt S, Søvik TT, Aasheim ET, et al. Secondary hyperparathyroidism, vitamin D sufficiency, and serum calcium 5 years after gastric bypass and duodenal switch. Obes Surg. 2013;23(3):384-90.

7. Christakos S, Dhawan P, Verstuyf A, et al. Metabolism, molecular mechanism of action, and pleiotropic effects. Physiol Rev. 2016;96(1):365-408.

8. Goldenberg D, Ferris RL, Shindo ML, et al. Thyroidectomy in patients who have undergone gastric bypass surgery. Head Neck. 2018;40(6):1237-44.

9. AlloMiguel G, García Fernández E,Martínez Díaz-Guerra G, et al. Recalcitrant hypocalcaemia in a patient with post-thyroidectomy hypoparathyroidism and Roux-en-Y gastric bypass. Obes Res Clin Pract. 2016;10(3):344-7.

10. Campos GM, Ziemelis M, Paparodis R, et al. Laparoscopic reversal of Roux-en-Y gastric bypass: technique and utility for treatment of endocrine complications. Surg Obes Relat Dis. 2014;10(1):36-43.

11. Droeser RA, Ottosson J, Muth A, et al. Hypoparathyroidism after total thyroidectomy in patients with previous gastric bypass. Langenbeck's Arch Surg. 2017;402(2):273-80.

12. Davis DB, Khoraki J, Ziemelis $\mathrm{M}$, et al. Roux en $\mathrm{Y}$ gastric bypass hypoglycemia resolves with gastric feeding or reversal: confirming a non-pancreatic etiology. Mol Metab. 2018;9:1527.

13. Goldfine AB, Mun EC, Devine E, et al. Patients with neuroglycopenia after gastric bypass surgery have exaggerated incretin and insulin secretory responses to a mixed meal. $\mathrm{J}$ Clin

Endocrinol Metab. 2007;92(12):4678-85.

14. McLaughlin T, Peck M, Holst $\mathrm{J}$, et al. Reversible hyperinsulinemic hypoglycemia after gastric bypass: a consequence of altered nutrient delivery. J Clin Endocrinol Metab. 2010;95(4):1851-5. 